\title{
Laser devices with stacked layers of InGaAs/GaAs quantum rings
}

\author{
Ferran Suárez, Daniel Granados, María Luisa Dotor and \\ Jorge M García \\ Instituto de Microelectrónica de Madrid, CNM (CSIC), C/Isaac Newton 8, PTM, \\ 28760-Tres Cantos (Madrid), Spain \\ E-mail: ferran@imm.cnm.csic.es
}

Received 30 September 2003

Published 29 January 2004

Online at stacks.iop.org/Nano/15/S126 (DOI: 10.1088/0957-4484/15/4/003)

\begin{abstract}
Stacked layers of $\mathrm{In}(\mathrm{Ga}) \mathrm{As}$ on $\mathrm{GaAs}(001)$ self-assembled quantum rings (QR) for laser application have been studied. Several samples with three stacked QR layers have been grown by molecular beam epitaxy with GaAs spacers from 1.5 to $14 \mathrm{~nm}$. The optical and structural properties have been characterized by photoluminescence spectroscopy and by atomic force microscopy, respectively. For GaAs spacers larger that $6 \mathrm{~nm}$, the stacked QR layers present similar properties to single QR layers. A semiconductor laser structure with three stacked layers of QR separated $10 \mathrm{~nm}$ in the active region has been grown. This spacer ensures well-developed rings with optical emission like that of a single layer. Laser diodes have been processed with 1-2 mm cavity lengths. The stimulated emission is multimodal, centred at $930 \mathrm{~nm}(77 \mathrm{~K})$, with a threshold current density per QR layer of $69 \mathrm{~A} \mathrm{~cm}^{-2}$. In this work, it is demonstrated that stacking rings is possible, and that a broad area laser with three $\mathrm{QR}$ layers can be fabricated successfully.
\end{abstract}

(Some figures in this article are in colour only in the electronic version)

\section{Introduction}

During last few years, many efforts have been devoted to the fabrication of optoelectronic devices based on low dimensional semiconductor nanostructures. Significant improvements of the laser performance for optical communication systems have already been demonstrated, such as: low threshold current [1$3]$, temperature-insensitive operation $[4,5]$, large modulation bandwidth, low chirp and linewidth enhancement factor [6]. Self-assembled semiconductor quantum dots (QD), formed via lattice-mismatched heteroepitaxy (for example InAs/GaAs, $\mathrm{InAs} / \mathrm{InP}, \mathrm{GaSb} / \mathrm{GaAs}$ ), are currently being used for developing optoelectronic technologies and devices. A precise control over the QD height, width and shape is of crucial importance since these structural properties have a clear influence on the quantum confinement of the charge carriers and therefore determine their optoelectronic properties. Unfortunately, self-assembled dots do not have the flexibility of band gap engineering that quantum wells have. Commonly the size of the dots is critically determined by the optimal growing conditions employed to obtain narrow size distributions. In the particular case of InAs on $\mathrm{GaAs}(001) \mathrm{QD}$, on which this work is focused, employing a different substrate temperature or changing the III/V element ratio may produce variations in the sizes of the dots, but also broadens the size distribution. This is one of the major problems if we aim to employ ensembles of these nanostructures in useful devices. A possible strategy for overcoming this problem consists in growing the QD under optimal conditions and modifying its vertical size during capping, without changing the embedding layers. A technique which is specially useful for reducing the vertical size, i.e. for obtaining shorter wavelengths [7], consists in partially covering the InAs islands with a thin cap of GaAs and letting the original islands 'melt' and re-shape. This process can be used to reduce the original vertical size of the QD in a controlled way with atomic height accuracy using, for example, the molecular beam epitaxy (MBE) growing technique. In particular, under specific growth conditions $[8,9]$ it is possible to obtain from each QD a self-assembled 'nanovolcano', or quantum ring (QR) $[10,11]$. Unlike mesoscopic lithography-defined rings, 
these $\mathrm{QR}$, once embedded in a GaAs matrix, function in the true quantum limit, free of decoherence problems due to scattering processes [10]. The possibility of tailoring the potential confinement in each layer of dots is also of great significance if we aim to achieve a more flexible band gap engineering of 3D nanostructures [7, 12].

When QD are employed in the active region of laser devices, the use of stacked layers is necessary to avoid saturation gain effects. It has been shown [13] that the optical mode loss does not increase as the number of layers increase, whereas the modal gain obtainable from the dot ground state increases. When QR are employed in the active region, stacking the nanostructures is even more important due to the commonly low density of ring ensembles $\left(2-9 \times 10^{9} \mathrm{~cm}^{-3}\right)$. The use of higher QR sheet densities could lead to overlap problems because of the large in-plane dimension of the rings $(100 \mathrm{~nm} \times 90 \mathrm{~nm})$. Although stacking of rings is of interest in much applied and fundamental research, it is not a solved problem. A lot of experimental and theoretical work has been reported on single layers of rings, but, as far as we know, only one theoretical work [14] on stacked QR, predicting parity coupling effects, has been published. Vertical coupling between QD may also allow their use for information processing. For high power laser devices, the low density of rings could be a drawback, but for applications such as Q-bit implementation, employing these nanostructures could be especially appropriate.

In this work, it is demonstrated that stacking rings is possible, and that a broad area laser with three QR layers can be fabricated successfully. The paper is organized as follows. After the description of the experimental set-up, the sample growth, the layout of the laser epitaxy and the device processing technology (section 2), an optical and structural characterization of various samples with three stacked layers of QR will be shown in section 3. The results from this section allow us to chose the optimum GaAs spacer between rings to use in the active region of the laser. Section 4 is focused on discussion of the characterization at different temperatures of the laser device processed with various cavity lengths.

\section{Experimental set-up and sample growth}

All the samples have been grown and the laser epitaxy carried out in a home-made solid source MBE. Group III cells, an As-valved cell with a cracker, the sample transfer, the sample oven and the shutter operation have been designed and built at the Institute of Microelectronics of Madrid. Reflection high energy electron diffraction (RHEED) has been used to monitor the onset growth of the QD and to calibrate both temperatures and growth rates.

The emission characteristics have been measured by photoluminescence spectroscopy (PL) with a $514.5 \mathrm{~nm} \mathrm{Ar}$ laser line and a $0.22 \mathrm{~m}$ monochromator with a liquid nitrogen cooled Ge diode. The surface sample morphology has been characterized by contact mode atomic force microscopy (AFM).

Two sets of samples have been grown with three layers of QR, each sample with a different GaAs spacer between layers: $1.5,3,4.5,6,10$ and $14 \mathrm{~nm}$ (see the inset of figure 1). In each layer, the total amount of deposited InAs was just above the critical thickness $\left(\Theta_{\mathrm{c}}\right)$ necessary for the $3 \mathrm{D}$ transition

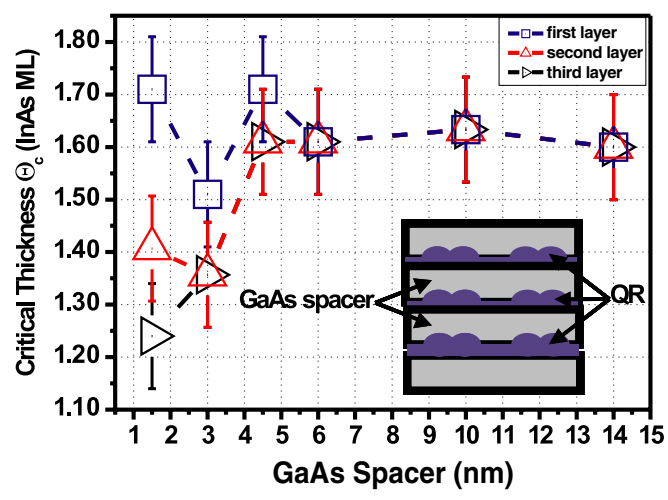

Figure 1. Critical thicknesses at which the QD nucleate in the first (square), second (triangles pointing up) or third (triangles pointing to the right) layer versus the GaAs spacer thickness. Inset: the scheme of a PL sample with three stacked layers of rings.

to take place (checked with RHEED). Commonly, $\Theta_{\mathrm{c}}$ is 1.6 ML for a single QD layer. Conversely, for 1.5 and $3 \mathrm{~nm}$ GaAs spacers, the nucleation of the second and the third layers takes place for coverage, $\Theta_{c}$, significantly below 1.6 ML. This effect is probably due to strong In segregation and the strain propagation effect, as occurs with stacked QD for thicker spacers; whereas for a spacer $4.5 \mathrm{~nm}$ thick, or thicker, the nucleation of the second and third dots takes place for 1.6 ML, as in the first layer. Studies on cross section structural microscopy have been performed, confirming that the ring-shaped islands remain when these nanostructures are overgrown with GaAs or stacked in different layers. These results will be presented in further publications.

The AFM samples have the top layer of rings uncovered and are cooled down immediately after growth and removed from the chamber to be characterized ex situ. The PL set of samples has been obtained by capping similar structures with a thick GaAs layer.

The laser epitaxy has been performed under continuous rotation using $\mathrm{As}_{4}$ for the whole structure, except for the ring layers where $\mathrm{As}_{2}$ has been used. Separate confinement heterostructures $(\mathrm{SCH})$ with $\mathrm{Si}$ and graded $\mathrm{Be}$ dopants have been used for the $\mathrm{n}^{+}$and $\mathrm{p}^{+}$cladding layers. The graded index waveguide (GRIN) has been grown with AlAs/GaAs short period superlattices. In the active region, three stacked layers of QR spaced by $10 \mathrm{~nm}$ of GaAs have been used. Full details of the layout for the laser epitaxy may be found in table 1 .

$\mathrm{Au}-\mathrm{Ge}-\mathrm{Au}$ and $\mathrm{Cr}-\mathrm{Au}$ have been used for the $\mathrm{n}$ and $\mathrm{p}$ contacts, respectively. Broad area lasers with $40 \mu \mathrm{m}$ wide stripes with cleaved front and rear facets have been fabricated with cavity lengths $(L)$ of $1,1.5$ and $2 \mathrm{~mm}$. Diodes were mounted with the p side up onto copper heat sinks. Devices were tested under pulsed conditions $(0.1 \%$ duty cycle) in a closed cycle temperature controlled helium cryostat. The spectra were analysed by a $0.22 \mathrm{~m}$ monochromator with an InGaAs photodetector.

\section{Single and stacked layers of quantum rings}

Figure 2(a) presents the AFM characterization of a selfassembled single layer of a typical InAs QD ensemble, grown under optimal conditions to obtain a narrow size distribution. 
Table 1. QR laser structure.

\begin{tabular}{|c|c|c|c|}
\hline \multicolumn{4}{|l|}{ Surface } \\
\hline$\overline{\text { Cladding }}$ & $\begin{array}{l}50 \mathrm{~nm} \\
400 \mathrm{~nm} \\
640 \mathrm{~nm} \\
320 \mathrm{~nm}\end{array}$ & $\begin{array}{l}\mathrm{GaAs} \\
\mathrm{Al}_{x} \mathrm{Ga}_{1-x} \mathrm{As} \\
\mathrm{Al}_{x} \mathrm{Ga}_{1-x} \mathrm{As} \\
\mathrm{Al}_{x} \mathrm{Ga}_{1-x} \mathrm{As}\end{array}$ & $\begin{array}{l}\text { Be doped }=1 \times 10^{19} \mathrm{~cm}^{-3} \\
\text { Be doped }=5 \times 10^{18} \mathrm{~cm}^{-3}, x=0.60 \\
\text { Be doped }=1 \times 10^{18} \mathrm{~cm}^{-3}, x=0.60 \\
\text { Be doped }=8 \times 10^{17} \mathrm{~cm}^{-3}, x=0.60\end{array}$ \\
\hline \multirow[t]{3}{*}{ Waveguide } & \multicolumn{3}{|c|}{$\begin{array}{l}82 \mathrm{~nm}, 29 \times[5 \mathrm{ML} \mathrm{GaAs}+5 \mathrm{ML} \mathrm{AlAs}], \text { equivalent to } \mathrm{Al}_{x} \mathrm{Ga}_{1-x} \mathrm{As}, x=0.50 \\
58 \mathrm{~nm}, 18 \times[7 \mathrm{ML} \mathrm{GaAs}+5 \mathrm{ML} \mathrm{AlAs}], \text { equivalent to } \mathrm{Al}_{x} \mathrm{Ga}_{1-x} \mathrm{As}, x=0.42 \\
56 \mathrm{~nm}, 14 \times[9 \mathrm{ML} \mathrm{GaAs}+5 \mathrm{ML} \mathrm{AlAs}], \text { equivalent to } \mathrm{Al}_{x} \mathrm{Ga}_{1-x} \mathrm{As}, x=0.36\end{array}$} \\
\hline & $\begin{array}{l}\text { Active } \\
\text { region }\end{array}$ & \multicolumn{2}{|c|}{$\begin{array}{l}10 \mathrm{~nm} \mathrm{GaAs} \\
2 \mathrm{ML} \mathrm{InAs}+2 \mathrm{~nm} \mathrm{GaAs} \text { (growth pause and ring formation) } \\
10 \mathrm{~nm} \mathrm{GaAs} \\
2 \mathrm{ML} \mathrm{InAs}+2 \mathrm{~nm} \mathrm{GaAs} \text { (growth pause and ring formation) } \\
10 \mathrm{~nm} \mathrm{GaAs} \\
2 \mathrm{ML} \mathrm{InAs}+2 \mathrm{~nm} \mathrm{GaAs} \text { (growth pause and ring formation) } \\
2.1 \mathrm{~nm} \mathrm{GaAs}\end{array}$} \\
\hline & \multicolumn{3}{|c|}{$\begin{array}{l}56 \mathrm{~nm}, 14 \times[9 \mathrm{ML} \mathrm{GaAs}+5 \mathrm{ML} \mathrm{AlAs}], \text { equivalent to } \mathrm{Al}_{x} \mathrm{Ga}_{1-x} \mathrm{As}, x=0.36 \\
58 \mathrm{~nm}, 18 \times[7 \mathrm{ML} \mathrm{GaAs}+5 \mathrm{ML} \mathrm{AlAs}], \text { equivalent to } \mathrm{Al}_{x} \mathrm{Ga}_{1-x} \mathrm{As}, x=0.42 \\
82 \mathrm{~nm}, 29 \times[5 \mathrm{ML} \mathrm{GaAs}+5 \mathrm{ML} \mathrm{AlAs}], \text { equivalent to } \mathrm{Al}_{x} \mathrm{Ga}_{1-x} \mathrm{As}, x=0.50\end{array}$} \\
\hline Cladding & $1400 \mathrm{~nm}$ & $\mathrm{Al}_{x} \mathrm{Ga}_{1-x} \mathrm{As}$ & Si doped $=1 \times 10^{18} \mathrm{~cm}^{-3}, x=0.60$ \\
\hline
\end{tabular}

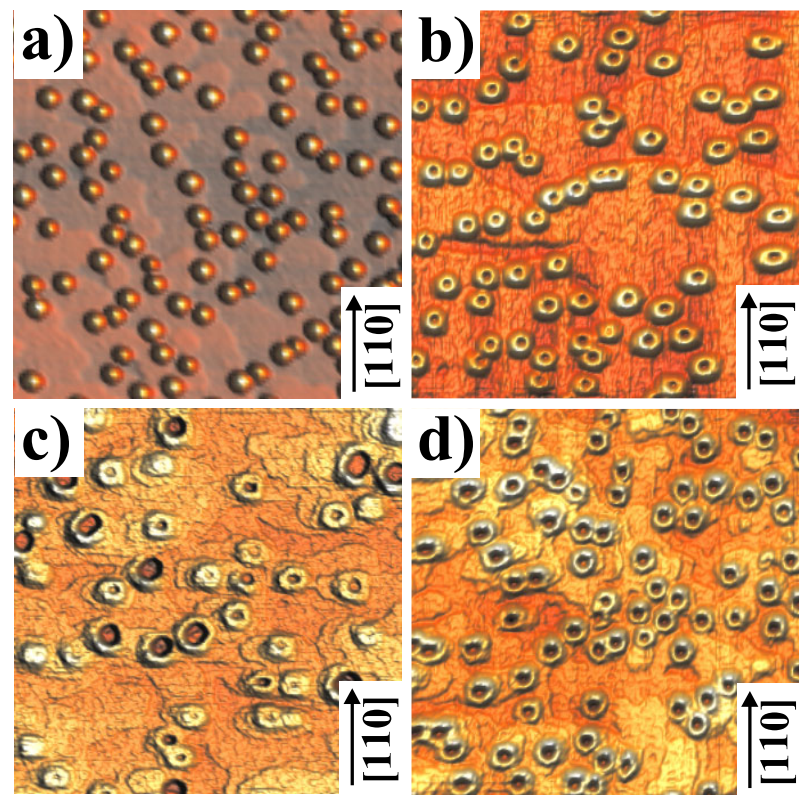

Figure $2.1 \times 1 \mu \mathrm{m}^{2}$ size AFM images of (a) a single layer of InAs/GaAs self-assembled QD, which are $11 \mathrm{~nm}$ high. When a growth pause is introduced during capping of QD with $2 \mathrm{~nm}$, each island re-shapes into a $\mathrm{QR}(\mathrm{b})$. Meanwhile when three $\mathrm{QR}$ are stacked with GaAs spacers of $3 \mathrm{~nm}$ (c) there are a variety of ring-like structures with no well-defined shape; for a $6 \mathrm{~nm}$ spacer (d), the surface morphology is very similar to that of a non-stacked sample of QR

The QR (figure 2(b)) were obtained after a growth interruption when the dots were partially covered with $2 \mathrm{~nm}$ of GaAs under an $\mathrm{As}_{2}$ flux, letting the material rearrange [15].

The AFM images of stacked QR samples are presented in figure 2(c) (spacer of $3 \mathrm{~nm}$ ) and figure 2(d) (spacer of $6 \mathrm{~nm}$ ). Figure 2(c) clearly shows that for a $3 \mathrm{~nm}$ spacer there are at least two families of ring-like structures. The bigger rings have a large hole which is at least (probably limited by the AFM tip resolution) $\sim 2.5 \mathrm{~nm}$ deep. Most probably, this hole is connecting a ring in the lower layer. The structure of these large rings suggests that a large mass transfer has occurred from the buried layer of $\mathrm{QR}$ to the top layer.

The surface morphology of stacked layers of rings with a spacer of $6 \mathrm{~nm}$ (figure $1(\mathrm{~d})$ ) is very similar to the one observed for a single layer of rings. For the set of samples with larger GaAs spacers (not shown), the top QR layer has always presented the same aspect, suggesting that the rings should have similar properties to a single layer.

Figure 3(a) shows the PL spectra at $15 \mathrm{~K}$ of a sample with a single layer of QD and of a sample with a layer of QR, both buried with GaAs. It should be underlined that the only difference between the two samples is that a growth interruption has been introduced for the ring sample when the dots were capped with $2 \mathrm{~nm}$ of GaAs. The amounts of deposited InAs are the same in both samples. A large shift in the emission wavelength from $1135 \mathrm{~nm}$ (QD) to $915 \mathrm{~nm}$ (QR) is observed. This large blue-shift $(\sim 260 \mathrm{meV})$ is due to a vertical reduction of the effective confining potential and it clearly demonstrates at-will tuning of the wavelength emission of the dots.

The similarity of properties of single and stacked layers of rings with spacers larger that $6 \mathrm{~nm}$, suggested by AFM analysis, has been confirmed by the optical properties analysis. The PL spectra at $15 \mathrm{~K}$ of three stacked layers of QR are presented in figure $3(\mathrm{~b})$. For 1.5 and $3 \mathrm{~nm}$ spacers, the spectra are red-shifted with respect to the single-layer emission. This effect can be understood on the basis of a strong vertical electronic coupling between the nanostructures with columnlike structures rather than isolated layers of rings. When a separation of $6 \mathrm{~nm}$ or more (not shown) was used between the layers of rings, the spectra were very similar to the one measured for a single layer.

These observations agree with the behaviour of the critical thickness required for QD formation $\left(\Theta_{c}\right)$ in each layer of the stacked rings (figure 1).

AFM characterization demonstrates that it is possible to grow stacked layers of QR using in each ring layer by the procedure described in [15]. For the fabrication with the laser, a $10 \mathrm{~nm}$ spacer between the layers of rings in the active region 


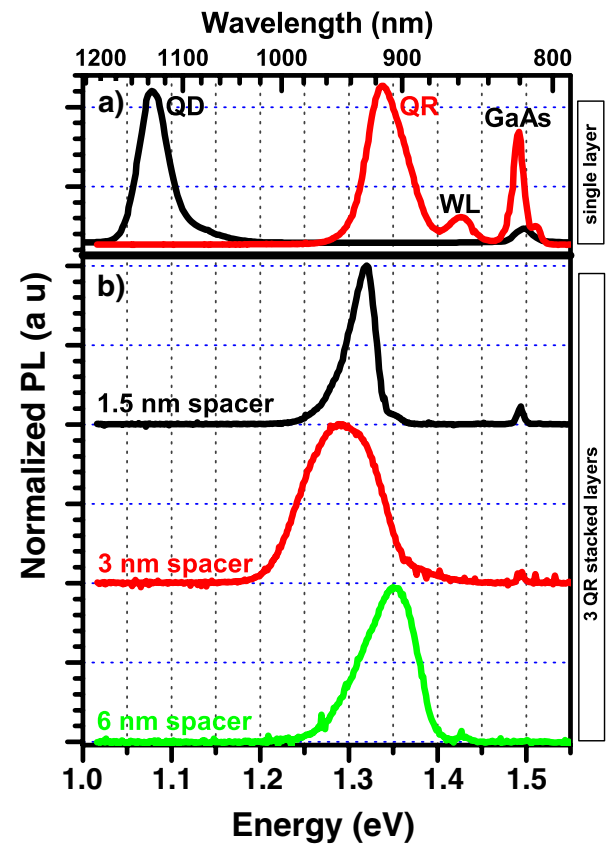

Figure 3. Low temperature PL spectra of (a) QD and a QR ensemble. A strong blue-shift in wavelength is observed, from dot to ring emission. (b) PL emission from samples with three stacked layers of $\mathrm{QR}$ with $1.5,3$ and $6 \mathrm{~nm}$ GaAs spacers.

has been chosen. This spacer will ensure well-developed rings with optical emission like that of a single layer.

\section{Laser characterization}

Devices with cavity lengths $(L)$ of $1,1.5$ and $2 \mathrm{~mm}$ have been tested under pulsed conditions. The light current characteristics for a $L=1 \mathrm{~mm}$ laser for a temperature range from 15 to $150 \mathrm{~K}$ are shown in figure 4 . The minimum measured value of the threshold current density $(J$ th) is $69 \mathrm{~A} \mathrm{~cm}^{-2}$ per QR layer sheet at $77 \mathrm{~K}$ for a device with a cavity length $L=2 \mathrm{~mm}$. No lasing emission is obtained above $150 \mathrm{~K}$. It is observed that the threshold current becomes smaller with $T$ decreasing from 150 to $77 \mathrm{~K}$ and, surprisingly, starts increasing below $77 \mathrm{~K}$. Such effects occur for all cavity lengths studied, as can be seen in figure 4 inset, and can be explained taking into account that the short period superlattices of the waveguide are not totally transparent for low temperature [15] - below $77 \mathrm{~K}$ in this particular case. This is confirmed by the observation with the naked eye of an additional spontaneous emission in the red spectral region for the devices studied. These radiative recombination processes (and probably other nonradiative ones) are due to the presence of carriers in the optical confinement layer, giving rise to an additional component of the injection current resulting in $T_{0}=-40 \mathrm{~K}$.

From 77 to $120 \mathrm{~K}, T_{0}$ is $80 \mathrm{~K}$, and for $T>120 \mathrm{~K}$ the $T_{0}$ value decreases to $40 \mathrm{~K}$. The low values of $T_{0}$ may be explained by the small vertical carrier confinement of the $\mathrm{QR}$, which are significantly shorter than the QD. Therefore, as the temperature is increased it is necessary to inject higher currents to maintain the threshold gain and compensate for the fraction of thermally evaporated carriers. At the same time, the volume of a QR active layer is one order (two orders) of magnitude smaller than

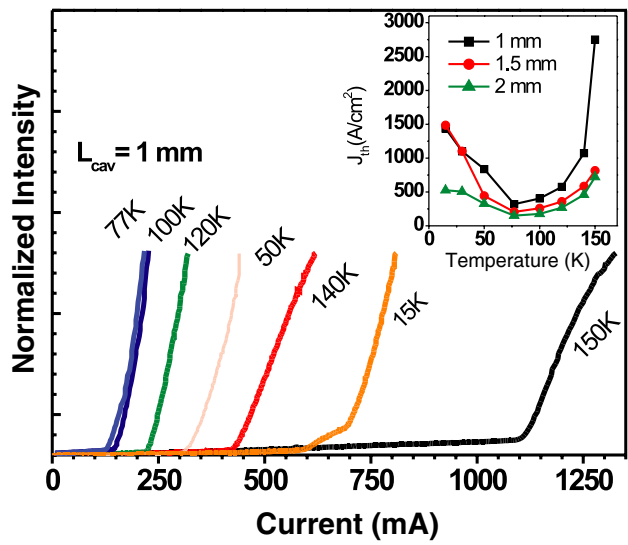

Figure 4. Light current characteristics of a $1 \mathrm{~mm}$ long $\mathrm{QR}$ laser at different temperatures. The inset shows the threshold current density versus $T$ for $1,1.5$ and $2 \mathrm{~mm}$ cavity lengths.

a QD (quantum well) active layer. So their optical confinement $(\Gamma)$ is also minor. The maximum expected gain (proportional to $\Gamma$ ) is low-becoming even lower as $T$ increases_-and this reduces the possibility of achieving a working laser device with three stacked layers of QR at room temperature. Even for a $2 \mathrm{~mm}$ cavity length, stimulated emission above $150 \mathrm{~K}$ has not been observed, suggesting gain saturation effects. The first way to overcome this limitation is to increase the active volume by stacking more than three layers of QR. Other approaches, used in order to obtain a temperature-insensitive QD laser, have been proposed based on band gap engineering. For example, the use of high confinement barriers next to the dots, in the active layer, will induce wide energy separations of electronic levels [16, 17]. Another method is based on selective tunnelling injection of carriers into the QD [18]. A novel approach recently published [19] is based on blocking the minority-carrier transport into the QD while leaving majoritycarrier injection unimpeded.

Figure 5 shows the multimodal stimulated spectra from the cleaved edge measured at $120,77,50$ and $15 \mathrm{~K}$ for $J$ th $=$ $1.6 \mathrm{~J}$ th. The laser emission clearly relates to recombination of carriers in the QR nanostructures, as can be deduced from its overlap with the QR PL broadband emission (see the inset of figure 5).

At $15 \mathrm{~K}$ there are measured two groups of multimode lines at 907 and $921 \mathrm{~nm}$, separated by $21 \mathrm{meV}$. The most intense mode emits at lower energy. The unexpected increment of the threshold current below $77 \mathrm{~K}$ with decreasing device temperature can be understood in terms of the loss of efficiency occurring because of the lack of transparency in the waveguide. Under high injection currents, the main mode operates together with the high energy lasing multimode.

\section{Conclusions}

In order to develop a QR laser device, stacked layers of QR have been studied for different GaAs spacers. We have seen proof that three stacked layers of QR are morphologically and optically similar to a single layer of QR for GaAs spacers thicker than $6 \mathrm{~nm}$. For this reason, GRIN SCH laser epitaxy was carried out with a $10 \mathrm{~nm}$ GaAs spacer between QR in the active layer. Laser emission using three stacked layers of QR 


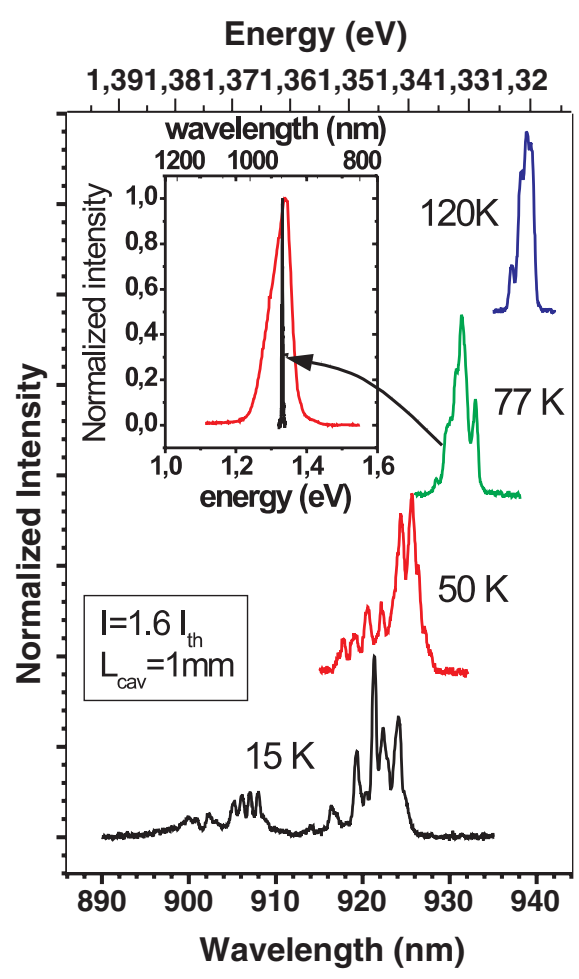

Figure 5. Lasing spectra of a $1 \mathrm{~mm}$ cavity laser at 120, 77, 50 and $15 \mathrm{~K}$. The inset shows the photoluminescence of three layers of QR (broad peak) and the laser emission (narrow peak) at $77 \mathrm{~K}$

in the active region was achieved for the first time, although working below $150 \mathrm{~K}$. The absence of complete transparency of the waveguide below $77 \mathrm{~K}$ explains the observed negative $T_{0}=-40 \mathrm{~K}$. The multimodal laser stimulated emission, centred at $930 \mathrm{~nm}$ at $77 \mathrm{~K}$, clearly relates to the recombination of carriers in the QR as can be deduced from the observation of the stimulated emission within the QR PL broadband. The minimum threshold current density per QR layer is $69 \mathrm{~A} \mathrm{~cm}^{-2}$ at $77 \mathrm{~K}$ for a $2 \mathrm{~mm}$ cavity length. The traces of gain saturation above $150 \mathrm{~K}$ suggest that the active volume is not enough to achieve stimulated emission; therefore it is necessary to stack more than three sheets of QR in order to develop a laser device working at RT.

\section{Acknowledgments}

This work was partially supported by Project No 07T/0062/ 2000 of the Comunidad Autónoma de Madrid, Project No TIC99-1035-C02, the European Commission Growth programme NANOMAT project, contract No G5RD-CT-200100545, and by Spanish MCYT under NANOSELF project TIC2002-04096-C03-03.

\section{References}

[1] Huffaker D L, Park G, Zou Z, Shchekin O B and Deppe D G $19981.3 \mu \mathrm{m}$ room-temperature GaAs-based quantum dot laser Appl. Phys. Lett. 732566

[2] Wang Z-G, Chen Y-H, Liu F-Q and Xu B 2001 Self-assembled quantum dots, wires and quantum-dot lasers J. Cryst. Growth 227/228 1132

[3] Sellin R L, Ribbat C, Bimberg D, Rinner F, Konstanzer H, Kelemen M T and Mikulla M 2002 High-reliability MOCVD-grown quantum dot laser Electron. Lett. 38883

[4] Pradhan S, Ghosh S and Bhattacharya P 2002 Temperature dependent steady-state characteristics of high-performance tunnel injection quantum dot lasers Electron. Lett. 381449

[5] Shchekin O B and Deppe D G $20021.3 \mu \mathrm{m}$ InAs quantum dot laser with $T_{0}=161 \mathrm{~K}$ from 0 to $80^{\circ} \mathrm{C} \mathrm{Appl.} \mathrm{Phys.} \mathrm{Lett.} 80$ 3277

[6] Zhang L and Boggess Thomas F 2000 Dynamic response of $1.3 \mu \mathrm{m}$ wavelength InGaAs/GaAs quantum dots Appl. Phys. Lett. 761222

[7] García J M, Mankand T, Holtz T, Wellmann P J and Petroff P M 1998 Electronic states tuning of InAs self-assembled quantum dots Appl. Phys. Lett. 723172

[8] García J M, Medeiros-Ribeiro G, Schmidt K, Ngo T and Petroff P M 1997 Intermixing and shape changes during the formation of InAs self-assembled quantum dots Appl. Phys. Lett. 712014

[9] García J M, Granados D, Silveira Juan P and Briones F 2004 Microelectronics 35 7-11

[10] Lorke A, Luyken R J, Govorov A O, Kotthaus J, García J M and Petroff P M 2000 Spectroscopy of nanoscopic semiconductor rings Phys. Rev. Lett. 842223

[11] Warburton R J, Pettersson H, Lorke A, Karrai K, Kotthaus J P, García J M and Petroff P M 2000 Optical emission from single, charge-tunable quantum rings Nature $\mathbf{4 0 5} 926$

[12] Granados D and García J M 2003 In(Ga)As self assembled quantum ring formation by molecular beam epitaxy Appl. Phys. Lett. 822401

[13] Smowton P M, Hermann E, Ning Y, Summers H D and Blood P 2001 Optical mode loss and gain of multiple-layer quantum-dot lasers Appl. Phys. Lett. 782629

[14] Ahn K-H and Peter F 2000 Parity effects in stacked nanoscopic quantum rings Phys. Rev. B 62 R4813 (Rapid communication)

[15] Dotor M L, Recio M, Golmayo D and Briones F 1992 Photoluminescence characterization of GaAs quantum well laser structure with AlAs/GaAs superlattices waveguide J. Appl. Phys. 725861

[16] Park G, Shchekin O B and Deppe D G 2000 Temperature dependence of gain saturation in multilevel quantum dot lasers IEEE J. Quantum Electron. 361065

[17] Groom K M, Tartakovskii A I, Mowbray D J, Skolnick M S, Smowton P M, Hopkinson M and Hill G 2002 Comparative study of InGaAs quantum dot lasers with different degrees of dot layer confinement Appl. Phys. Lett. 811

[18] Pradhan S, Ghos S and Bhattacharya P 2002 Temperature dependent steady-state characteristics of high-performance tunnel injection quantum dot lasers Electron. Lett. 381449

[19] Asryan Levon V and Luryi S 2002 Temperature-insensitive semiconductor quantum dot laser Solid State Electron. 47 205 